\title{
Decreased neuroautonomic complexity in men during an acute major depressive episode: analysis of heart rate dynamics
}

\author{
SJ-J Leistedt ${ }^{1}$, P Linkowski ${ }^{1}$, J-P Lanquart ${ }^{1}$, JE Mietus ${ }^{2}$, RB Davis $^{3}$, AL Goldberger ${ }^{2,4}$ and MD Costa ${ }^{2,4}$
}

Major depression affects multiple physiologic systems. Therefore, analysis of signals that reflect integrated function may be useful in probing dynamical changes in this syndrome. Increasing evidence supports the conceptual framework that complex variability is a marker of healthy, adaptive control mechanisms and that dynamical complexity decreases with aging and disease. We tested the hypothesis that heart rate (HR) dynamics in non-medicated, young to middle-aged males during an acute major depressive episode would exhibit lower complexity compared with healthy counterparts. We analyzed HR time series, a neuroautonomically regulated signal, during sleep, using the multiscale entropy method. Our results show that the complexity of the HR dynamics is significantly lower for depressed than for non-depressed subjects for the entire night $(P<0.02)$ and combined sleep stages 1 and $2(P<0.02)$. These findings raise the possibility of using the complexity of physiologic signals as the basis of novel dynamical biomarkers of depression.

Translational Psychiatry (2011) 1, e27; doi:10.1038/tp.2011.23; published online 26 July 2011

\section{Introduction}

Major depression (MD) is highly prevalent and a leading cause of disability worldwide. ${ }^{1-3} \mathrm{MD}$ is a multisystem illness, affecting, for example, endocrine, immunological, nocioceptive and cardiovascular function. ${ }^{4,5}$ Patients have a twofold to fourfold increased risk of developing cardiovascular disease ${ }^{6}$ and of mortality after a cardiac event. ${ }^{7}$ One underlying mechanism could be related to dysregulation of the autonomic nervous system. ${ }^{8,9}$ However, the diagnosis of MD still lacks objective assays or genomic markers. ${ }^{10-12}$

To the extent that this syndrome affects multiple regulatory mechanisms operating over a wide range of time scales, diagnostic and therapeutic approaches might be enhanced by (i) probing signals that reflect integrated physiology, such as heart rate variability (HRV) ${ }^{13,14}$ and (ii) using newer measures that quantify dynamical properties on multiple time scales. ${ }^{15-17}$

Beat-to-beat heart rate (HR) fluctuations may be particularly relevant to the understanding of the pathophysiology of MD as they encode information about underlying neuroautonomic control. ${ }^{13,14}$ Previous reports ${ }^{18-23}$ on HR dynamics in $\mathrm{MD}$ generally suggest decreased parasympathetic or increased sympathetic effects. However, the findings are not fully consistent and discrepancies are difficult to resolve because of the heterogeneity of the populations studied, confounding medication effects and differences in analytical tools.
Here, we studied a population of non-medicated, young to middle-aged men during an acute MDE and a healthy control group. We quantified the complexity of cardiac interbeat interval time series, ${ }^{15-17}$ for the entire night and different sleep stages, to gain further insight into the effects of MDE on HR dynamics.

Complexity relates to the information content (structural 'richness') of a signal ${ }^{24}$ that emerges from the nonlinear interactions among regulatory components. Two signals may have identical variance but different complexity values that reflect differences in their dynamical properties.

High multiscale complexity has been proposed as a generic feature of healthy dynamics. ${ }^{25}$ In contrast, disease and aging, marked by degraded functionality and adaptability, are characterized by loss of complexity. ${ }^{15,17,26,27}$ This study was aimed at testing the hypothesis that MDE is associated with degradation in the complexity of HR dynamics, reflecting neuroautonomic perturbations.

Complexity of cardiac interbeat interval fluctuations during sleeping hours was assessed using the multiscale entropy (MSE) method described in detail elsewhere. ${ }^{15,17}$ This method has been widely used in the analysis of physiologic time series. ${ }^{28-30}$ MSE quantifies the complexity of a signal by computing an entropy measure called sample entropy ${ }^{31,32}$ (SampEn) on different time scales. Of note, conventional single scale-based entropy and mutual information algorithms

\footnotetext{
${ }^{1}$ Sleep Laboratory, Psychiatric Laboratory Research, Psychiatric Department, Erasme Academic Hospital, Free University of Brussels (ULB), Brussels, Belgium; ${ }^{2}$ Division of Interdisciplinary Medicine and Biotechnology, Beth Israel Deaconess Medical Center, Harvard Medical School, Boston, MA, USA; ${ }^{3}$ Division of General Medicine Primary Care, Beth Israel Deaconess Medical Center, Boston, MA, USA and ${ }^{4}$ The Wyss Institute for Biologically Inspired Engineering at Harvard University, Boston, MA, USA

Correspondence: Dr MD Costa, Division of Interdisciplinary Medicine and Biotechnology, Beth Israel Deaconess Medical Center, Harvard Medical School, 330 Brookline Avenue, Boston, MA 02215, USA.

E-mail: mcosta3@bidmc.harvard.edu

Keywords: complexity; depression; heart rate variability; nonlinear dynamics

Received 26 May 2011; accepted 1 June 2011
} 
are irregularity measures, which yield higher values for uncorrelated random signals than for intrinsically complex ones. In contrast, MSE analysis reveals that time-varying signals such as cardiac interbeat intervals under healthy conditions are more complex than highly irregular ones generated by pathologic processes such as atrial fibrillation.

In this study, we focused on the analysis of nocturnal HR time series as (i) a continuous electrocardiogram (ECG) is routinely recorded with polysomnography (PSG) and (ii) the level of physical activity among sleeping subjects is likely more comparable than during waking hours.

In addition to the complexity analysis, we also present conventional HRV analyses comprising time and frequency (Fourier-based) domain measures. ${ }^{33}$ The latter require the data to be stationary and linear. Despite the fact that physiologic data are almost invariably non-stationary and non-linear, traditional HRV measures are among the most frequently computed. Therefore, we provide them supplementarily, further noting that they are not directly relevant for the hypothesis that we test.

\section{Subjects and methods}

Subjects. A total of 20 control subjects and 25 unmedicated male inpatients with an acute episode of MD, according to DSM-IV-TR criteria, enrolled this study (Table 1). Healthy controls were recruited from the community; patients were recruited from both the Sleep Laboratory and an inpatient psychiatry ward at Erasme Academic Hospital in Brussels. Controls were determined to be free of DSM-IV-TR axis I or axis II diagnoses and had no family history of major psychiatric disorders. Subjects reported a regular sleepwake schedule and no current or past sleep disorders. Depressive symptom severity was assessed with the 24-item Hamilton rating scale for depression. ${ }^{34}$

Patients were included if they had the following: (1) a Hamilton rating scale for depression score of 20 or greater; ${ }^{34}$ (2) were not taking psychotropic medications and (3) had a Pittsburgh Sleep Quality Index of five or greater. ${ }^{35}$ Subjects were excluded if they had untreated or poorly controlled conditions known to confound analysis of the sleep electroencephalogram and/or HRV results, for example, diabetes mellitus and chronic heart failure.

In addition, we also excluded subjects who required treatment with agents known to affect either signal (for example, $\beta$-blockers or corticosteroids). Both controls and patients were medically screened with complete physical examination, chest X-ray, 12-lead ECG, electroencephalography and standard laboratory tests as well as blood and urine toxicology screens. They did not show evident cardiovascular or endocrine abnormalities, primary sleep disorders and had a body mass index $<29 \mathrm{~kg} \mathrm{~m}^{-2}$. Before signing informed consent, each subject received a detailed description of the procedures involved in the study and was deemed capable. The protocol was approved by the Ethics Committee of Erasme Academic Hospital, Free University of Brussels. A more complete description of our recruitment procedures is provided elsewhere. ${ }^{36}$

Recordings and experimental conditions. In the patient group, sleep studies were conducted after at least a 3-week, psychotropic medication-free evaluation period. PSG recordings were obtained during two to three consecutive nights, of which, only the last one was examined in this study. ${ }^{37}$ HR parameters were obtained from analysis of cardiac interbeat intervals from the continuous ECG recorded as part of the PSG exams, (Alice 5 Diagnostic Sleep System, Philips Respironics, Murrysville, PA, USA). Nineteen lead electroencephalograms were recorded according to the International 10-20 Standard, with a contralateral reference to the A1 or A2 mastoid derivation, along with two electrooculograms and one submental electromyogram, as previously described. ${ }^{36}$ Using a customized program (Endymion 1993-2009, Sleep Laboratory, Erasme Hospital) to facilitate analysis, each 20-second PSG epoch was visually scored according to standard criteria. $^{38}$

HRV computations. From the European data format (edf) files, the ECG signal was extracted and converted to open source WFDB format (http://www.physionet.org). An automated QRS detection algorithm was then used to detect beats and annotate them as either normal sinus or ectopic. ${ }^{39}$ Outliers due to missed or false beat detections were identified using a sliding window average filter. Intervals $<0.4$ s or greater than $2.0 \mathrm{~s}$ were excluded from the window average. Next, using a window of 41 intervals, the average over the window was calculated, excluding the central interval. If the central interval was outside $20 \%$ of the window average this interval was excluded and the window advanced by one interval.

Table 1 Selected demographic and clinical data

\begin{tabular}{lccc}
\hline & Healthy $(\mathbf{N}=\mathbf{2 0})$ & Depressed $(\mathbf{N}=\mathbf{2 5})$ & $\boldsymbol{P}$-value \\
\hline $\begin{array}{l}\text { Demographic data } \\
\text { Age (years) }\end{array}$ & $36(25-52)$ & $39(19-55)$ & 0.43 \\
Body mass index $\left(\mathrm{kg} \mathrm{m}^{-2}\right)$ & $23.7(21.1-28.1)$ & $22.2(17.1-28.0)$ \\
Clinical data & & & 0.17 \\
24-item Hamilton depression score & $1(0-3)$ & $32(24-51)$ \\
Pittsburgh sleep quality index & $1(1-6)$ & $10(7-20)$ & $<0.0001$ \\
\hline
\end{tabular}

Results are given as median and (minimum-maximum) range. 
From the resulting beat annotation files, we calculated the following standard time domain HRV statistics: the average of all the normal sinus to normal sinus (NN) intervals (AVNN), the s.d. of all NN intervals (SDNN), the s.d. of the averages of NN intervals in all 5-min segments (SDANN), the mean of the s.d.'s of NN intervals in all 5-min segments (SDNNINDX), the root mean square of consecutive differences between adjacent NN intervals ( $\mathrm{rMSSD}$ ), and the percentage of adjacent intervals whose difference is higher than $10 \mathrm{~ms}$ (pNN10), $25 \mathrm{~ms}$ (pNN25) and $50 \mathrm{~ms}$ (pNN50) ${ }^{40}$ (http:// www.physionet.org).

The following standard frequency domain measures were calculated using the Lomb periodogram for unevenly sampled data: total spectral power (TOTPWR; 0-0.4 Hz), ultra-low frequency power (ULF; 0-0.003 Hz), very-low frequency power (VLF; $0.003-0.04 \mathrm{~Hz}$ ), low frequency power (LF; $0.04-0.15 \mathrm{~Hz})$, high frequency power $(\mathrm{HF} ; 0.15-0.4 \mathrm{~Hz})$, the ratio of low to high frequency power (LF/HF) and the slope $(\beta)$ of the spectrogram on a log-log scale assessed over the range of 0 to $0.04 \mathrm{~Hz}$.

SDNN and TOTPWR are measures of variance. SDANN is a measure of the degree of non-stationarity of the time series. SDNNINDX quantifies how much the variance changes over time. rMSSD and pNN measures quantify HF fluctuations. Therefore, these time domain measures, in addition to HF power, have been used as indexes of cardiac vagal tone modulation. LF power is thought to reflect both sympathetic and vagal influences. LF/HF ratio has been proposed as an index of cardiac autonomic control but it is no longer widely accepted as a direct indicator of 'sympatho-vagal balance.' ULF and VLF powers quantify nonspecific trends in the time series. The $\beta$ exponent is one measure of the fractal scaling properties of a signal. For HR time series obtained from healthy subjects under baseline conditions, this exponent is usually close to -1 , which indicates the presence of longrange correlations. ${ }^{41,42}$

Complexity analysis. In order to quantify the dynamical complexity of the RR interval time series, we used the MSE method described in detail elsewhere. ${ }^{15,17}$ Briefly, the MSE method quantifies the degree of irregularity of a signal using an entropy measure, such as SampEn, over multiple time scales. SampEn ${ }^{31}$ is the negative natural logarithm of an estimate of the conditional probability that subseries (epochs) of length $m$ that match pointwise within a tolerance $r$ will also match when the length of each of these subseries increases from $m$ to $m+1$ data points. Signals that are highly irregular, and therefore more entropic, over a wide range of scales are more complex than both those that are highly regular, that is, periodic, and those that are irregular only at a single time scale (for example, white noise). The line obtained by connecting the SampEn values ( $y$ axis) for a range of time scales ( $x$ axis) is called the MSE curve. We then derived a short-term and longer-term complexity index $(\mathrm{Cl})$.

As noted, in traditional HRV analysis, the cutoff separating the low and high frequency bands is $0.15 \mathrm{~Hz}$, which corresponds to a period of $\sim 7 \mathrm{~s}$. Physiologically, the high frequency band encompasses HR fluctuations associated with respiration. To quantify the complexity of the dynamics over a comparable high-frequency band, we define a shortterm $\mathrm{Cl}$ as the area under the MSE curve ranging from scales 1 to 8 , inclusive (Given the fact that the average HR for both groups was about 60 beats per minute, the mean RR interval was $\sim 1 \mathrm{~s}$ and, therefore, scale $n$ corresponds to $\sim n$ seconds). We note that the specific cutoffs for both traditional frequency and complexity analyses are somewhat arbitrary.

To probe the complexity of the dynamics on relatively longer time scales, we computed a $\mathrm{Cl}$ defined as the area under the MSE curve from scales 1 to 20 . The upper cutoff scale chosen (20), although necessarily arbitrary, is based on previous studies of HR time series. ${ }^{17,30}$ Standard parameter values for calculating SampEn are as follows: $m=2$ and $r=15 \%$ of the time series' s.d.'s. ${ }^{31,32}$ Here, we chose $m=2$ and $r=8 \mathrm{~ms}$ for the following reason. The parameter $r$ determines the level of noise accepted. If $\left|x_{i}-x_{i+1}\right|>r$, then the two data points, $x_{i}$ and $x_{i+1}$, are distinguishable. If instead, $\left|x_{i}-x_{i+1}\right| \leqslant r$, the two data points are indistinguishable, that is, their difference is considered noise, not signal. As the ECG recordings were sampled at $250 \mathrm{~Hz}$, each RR interval is determined with an uncertainty of $4 \mathrm{~ms}(1 / 250)$, and the difference between RR intervals with an uncertainty of twice this value. To be above the noise level we chose $r=8 \mathrm{~ms}$. Qualitatively similar results were obtained using $r=15 \%$ of the time series' s.d.'s, corresponding to $r$ values ranging from 6 to $18 \mathrm{~ms}$.

The number of time scales one can probe depends on the total length of the original signal. Analysis of larger time scales requires longer signals. As a rule of thumb, ${ }^{32}$ at least 200 consecutive data points are needed for reliable calculation of SampEn. Therefore, if $N$ is the length of a signal and $S$ the largest time scale to be included in the MSE analysis, the relationship between the two variables should be such that $N / S>200$. For the analysis of HR dynamics during different sleep stages, we considered 15 min or longer segments with at least 1000 data points, and computed SampEn for time scales 1 to 5 , inclusive. The analysis of full-night HR dynamics is not constrained by the length of the time series, which ranged from about 23000 to 40000 data points. Therefore, in this case, we were able to compute a $\mathrm{Cl}$ encompassing entropy values for both short ( 1 to 8 ) and longer (1 to 20 ) time scales.

Biostatistical analyses. Mann-Whitney non-parametric $U$-tests were used to examine group (controls versus depression) differences in clinical, demographic, and sleep measures as well. This test was deemed most appropriate because of the sample size and data distribution. To adjust for age, we also fit least squares regression models. To evaluate the association between MSE and depression during specific sleep stages, we fit linear regression models. Because there were multiple observations per participant, we used generalized estimating equations methods with an exchangeable correlation structure to account for within-participant correlation. Spearman correlation was used to assess the association between MSE and the Hamilton depression scale among the depressed patients. All analyses were performed with a (type I error) set at 0.05 using the SAS statistical software (version 9.13 for Windows, SAS Institute, Cary, NC, USA). 


\section{Results}

Clinical data. Selected demographic and clinical data are summarized in Table 1.

PSG analyses. Standard PSG measures are summarized in Table 2. Consistent with previous reports, ${ }^{43,44}$ the depression versus healthy groups showed statistically significant decreases in sleep efficiency, total sleep time, rapid eye movement latency, percentage delta sleep, and increases in sleep onset latency, percentage stage 1 sleep, awakenings throughout the night and in rapid eye movement density.

Standard HRV analyses. Standard HRV measures are presented in Table 3. Depressed patients showed significantly decreased values for the following: (1) the mean of the heartbeat interval (AVNN); (2) the mean of the s.d.'s of NN intervals in all non-overlapping 5-min segments (SDNNINDX); (3) pNN10; (4) spectral power in the VLF and LF ranges. However, multiple other commonly used measures, including the s.d. of the NN intervals (SDNN) and high frequency power (HF), were not statistically different.

Complexity analysis of HR dynamics. SampEn of the RR interval time series derived from full-night ECG recordings was higher for the healthy than for the depressed subjects across all measured time scales (Figure 1). The $\mathrm{Cl}$ for short time scales, defined as the area under the MSE curve from scales 1 to 8 (corresponding to frequencies between 0.12 and $0.5 \mathrm{~Hz})$, was significantly $(P<0.04)$ higher for healthy

Table 2 Electroencephalographic sleep data summaries

\begin{tabular}{|c|c|c|c|}
\hline & Healthy $(N=20)$ & Depressed $(N=25)$ & P-value \\
\hline \multicolumn{4}{|l|}{ Sleep continuity } \\
\hline Total sleep time (min) & $487(436-543)$ & $375(204-485)$ & $<0.0001$ \\
\hline Sleep latency (min) & $28(7-40)$ & $48(19-186)$ & $<0.0001$ \\
\hline Sleep efficiency (\%) & $85.6(78.2-94.5)$ & $66.9(35.8-89.3)$ & $<0.0001$ \\
\hline No. of awakenings & $57(27-90)$ & $74(29-115)$ & 0.07 \\
\hline Awake time (min) & $58.5(14-90)$ & $98(38-204)$ & $<0.0001$ \\
\hline Awake time (\% SPT) & $10.4(3.2-16.4)$ & $22.1(13.7-47.0)$ & $<0.0001$ \\
\hline \multicolumn{4}{|l|}{ NREM } \\
\hline$\%$ Stage 1 & $7.9(3.8-11)$ & $10(2.9-61)$ & 0.004 \\
\hline$\%$ Stage 2 & $58.3(35.1-73.0)$ & $58(7.0-74.1)$ & n.s. \\
\hline$\%$ Delta & $14.1(7-25.9)$ & $3.8(0-17)$ & $<0.0001$ \\
\hline \multicolumn{4}{|l|}{$R E M$} \\
\hline$\%$ REM & $21.3(14.5-27.4)$ & $20.5(9.5-32.1)$ & n.s. \\
\hline$\%$ REM latency & $85.5(65-147)$ & $48(5-88)$ & $<0.0001$ \\
\hline$\%$ REM density (unit/min) & $2.1(1.3-3.2)$ & $4.8(2.5-7.4)$ & $<0.0001$ \\
\hline
\end{tabular}

Abbreviations: NREM, non-rapid eye movement; n.s., nonsignificant; REM, rapid eye movement; SPT, sleep period time. Results are indicated as median and (minimum-maximum) range.

Table 3 Heart rate variability analysis

\begin{tabular}{|c|c|c|c|}
\hline & Healthy $(N=20)$ & Depressed $(N=25)$ & P-value \\
\hline AVNN (sec) & $1.04(0.82-1.22)$ & $0.94(0.81-1.29)$ & 0.002 \\
\hline SDNN (sec) & $0.10(0.06-0.17)$ & $0.10(0.06-0.14)$ & n.s. \\
\hline SDANN (sec) & $0.07(0.04-0.12)$ & $0.06(0.04-0.10)$ & n.s. \\
\hline SDNNINDX (sec) & $0.08(0.05-0.11)$ & $0.06(0.03-0.10)$ & 0.02 \\
\hline rMSSD (sec) & 0.05 (0.02-0.09) & $0.04(0.01-0.09)$ & n.s. \\
\hline pNN10 & $0.82(0.59-0.89)$ & $0.73(0.14-0.89)$ & 0.05 \\
\hline pNN25 & $0.59(0.22-0.76)$ & $0.45(0.01-0.75)$ & n.s. \\
\hline pNN50 & $0.25(0.02-0.54)$ & $0.15(0.00-0.51)$ & n.s. \\
\hline TOTPWR $\left(\sec ^{2}\right)$ & $0.013(0.004-0.035)$ & $0.010(0.004-0.022)$ & n.s. \\
\hline ULF $\left(\sec ^{2}\right)$ & $0.006(0.001-0.019)$ & $0.004(0.002-0.012)$ & n.s. \\
\hline VLF $\left(\sec ^{2}\right)$ & $0.004(0.001-0.009)$ & $0.003(0.001-0.007)$ & 0.01 \\
\hline $\operatorname{LF}\left(\sec ^{2}\right)$ & $0.002(0.001-0.004)$ & $0.001(0.000-0.003)$ & 0.04 \\
\hline $\mathrm{HF}\left(\sec ^{2}\right)$ & $0.001(0.000-0.003)$ & $0.001(0.000-0.003)$ & n.s. \\
\hline LF/HF & $1.590(0.646-4.076)$ & $1.981(0.431-11.18)$ & n.s. \\
\hline$\beta$ exponent & $-0.94(-0.74$ to -1.28$)$ & $-0.92(-0.75$ to -1.15$)$ & n.s. \\
\hline
\end{tabular}

Abbreviations: AVNN, average of all the normal sinus to normal sinus intervals; HF, high frequency; LF, low frequency; n.s., nonsignificant; pNN10, percentage of adjacent intervals whose difference is higher than $10 \mathrm{~ms}$; pNN25, percentage of adjacent intervals whose difference is higher than $25 \mathrm{~ms}$; pNN50, percentage of adjacent intervals whose difference is higher than $50 \mathrm{~ms}$; rMSSD, root mean square of consecutive differences between adjacent NN intervals; SDANN, s.d. of averages of NN intervals in all 5-min segments; SDNN, s.d. of all NN intervals; SDNNINDX, mean of the s.d. of NN intervals in all 5-min segments; TOTPWR, total spectral power; ULF, ultra-low frequency; VLF, very-low frequency.

Results are indicated as median and (minimum-maximum) range. 


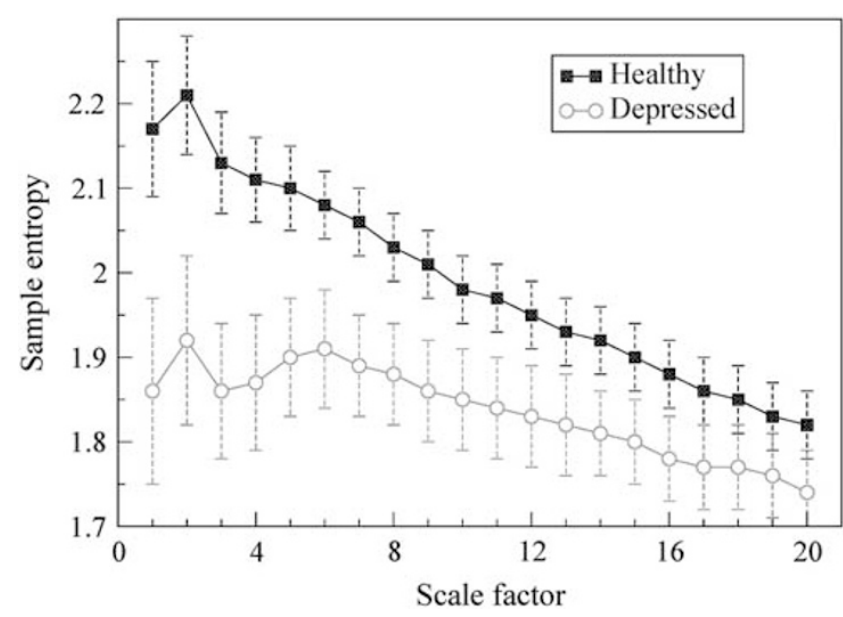

Figure 1 Multiscale entropy analysis of heart rate dynamics in men during a major depressive episode (open circles) and healthy controls (dark squares) for the full night. For all time scales displayed, the entropy values for the control group were higher than for the depression group, indicating that the heart rate dynamics of healthy subjects are more complex than those of depressed patients. The values are mean \pm s.e.

(median: 17.6; range: 13.8-19.4) than depressed (median: 15.4; range: 6.9-19.8) subjects. In a regression model adjusted for age, the $\mathrm{Cl}$ for depressed subjects was, on average, 1.8 (95\% confidence interval 0.3 to $3.3, P=0.02)$ lower than for healthy subjects.

Although the difference between the two groups is most apparent across the shorter time scales, the healthy group still trended ( $P=0.09$ unadjusted, $P=0.06$ adjusted for age) to higher dynamical HR complexity when longer time scales ( 1 to 20) were included in the analysis (Figure 1). To ascertain that these findings were not due to differences in the number of arousals and disrupted sleep architecture in MD, we computed HR complexity during different sleep states. This sub-analysis was restricted to continuous ECG segments $15 \mathrm{~min}$ or longer because of the minimum data length requirements for multiscale signal analysis (see Subjects and methods). In an effort to maximize the number of subjects included in this sub-analysis, we combined sleep stages 1 and 2 , and 3 and 4.

Only 8 out of $25(<30 \%)$ depression patients and 10 out of 20 healthy subjects had at least one 15-min or longer rapid eye movement segment. Similarly, only 10 depressed and 9 healthy subjects ( $<50 \%$ ) had at least one 15-min or longer segment of combined sleep stages 3 and 4, precluding the use of complexity analysis for these stages. Time series of sufficient length for multiscale analysis were available in 20 healthy and 23 depressed subjects during combined sleep stages phases 1 and 2 . For these non-rapid eye movement sleep periods, HR complexity was significantly higher for the control (median: 17.0; range: 7.7-30.3) than for the depression (median: 14.6; range: 9.1-28.0) group (difference of $1.43,95 \%$ confidence interval $0.38-2.48, P=0.007$, based on the generalized estimating equation model). Among depressed subjects, the $\mathrm{Cl}$ was inversely correlated with the Hamilton rating scale for depression score $(r=-0.40$, $P=0.05)$.

\section{Discussion}

The major finding of this study is that acute MDEs in young to middle-aged men are associated with a decrease in cardiac interbeat interval complexity during sleeping hours (Figure 1). This finding is consistent with a degradation in multiscale neuroautonomic regulation of $\mathrm{HR}$ over relatively short time scales ( $<20 \mathrm{~s}$ ). Further, lower $\mathrm{Cl}$ s tend to be associated with more severe depression (higher Hamilton rating scale for depression score). Our observations cannot be attributed to differences in medication, age, gender, activity or sleep architecture.

Two previous studies have sought to examine changes in HR complexity with depressive illness using MSE. In one ${ }^{45}$ the authors analyzed only daytime time series derived from relatively short $(30 \mathrm{~min})$ continuous ECG recordings and found that MSE for only one time scale $(\sim 3 s)$ was significantly higher for healthy subjects than for depressed patients. Our results differ importantly because (i) we analyze night-time recordings and (ii) we show a loss of complexity across a relatively broad range of time scales, not just a single one, which is a central requisite of the complexity-loss hypothesis.

Another study ${ }^{46}$ assessed HR complexity during both awake and 'bedtime' hours in a group of patients with major depressive disorder, a group with primary insomnia and a group of healthy controls. The authors reported a reduction in complexity during sleeping but not daytime hours for both patient groups. One limitation of this study is the fact that it did not include PSG recordings and, therefore, did not control for sleep stage effects. However, these results on MD syndrome are not inconsistent with the findings reported here for patients during a major depressive episode.

Boettger et al. ${ }^{47}$ reported on two different, putative measures of complexity in MD, including the scaling exponent $\beta$, a fractal dimension. In contrast with this study, we observed values of this exponent (Table 3 ) that were quantitatively consistent with previous reports for young adults ${ }^{41,42}$ but no statistical difference between depressed and non-depressed groups.

Our findings are also of potential interest because they are aligned with the emerging concepts ${ }^{16,17,25}$ that (1) the dynamics of healthy systems, which show the highest adaptability, functionality and reserve, are the most complex, and (2) a variety of pathologic states, ${ }^{28-30}$ as well as advanced aging, ${ }^{48,49}$ are associated with loss of dynamical complexity (information content). Our findings place MDE in this growing class of pathologic states characterized by a loss of complexity. The possible universality of complexity loss with pathology and aging may limit its diagnostic specificity. However, to the extent that complexity is a dynamical 'assay' of integrative (global) dynamics, measures such as MSE that probe multiscale features may provide novel ways to monitor individual patients over time and assess their response to therapeutic interventions intended to enhance functionality and plasticity.

We note that HR complexity is not directly related to traditional HRV as assessed by measures of variance, by spectral power in selected bands, or by spectral scaling exponents. Instead, MSE probes properties of the system that relate to the temporal structure of the signals it generates and 
not simply to the amplitude of their fluctuations. In this study, measures most directly related to the s.d. of HR time series from healthy subjects were actually comparable to those of depressed subjects (Table 3). Our findings suggest that a key difference between the cardiac interbeat interval time series from healthy and depressed patients appears to reside in their temporal correlations, that is, the organization across, not just within, a given time scale.

In a previous study ${ }^{17}$ of HR dynamics that included healthy young and elderly subjects, as well as patients with heart disease, we found that a monotonic decrease in HR entropy with time scale during night time was characteristic of both healthy young and healthy elderly subjects, but not of patients with heart disease. What distinguished the former two groups was the fact that the entropy values were significantly higher for the young than for the elderly. Of further interest is the finding in the present study that the MSE profiles for the healthy and depression groups (Figure 1) showed a monotonic decrease of entropy with time scale, qualitatively similar to the previously noted age-related changes in the MSE curves. These results, in conjunction with the fact that the MSE values were lower for the depression than the healthy group, support the putative link between the dynamics of aging and depression. ${ }^{50-52}$ The results are also in accord with evidence that severe mood disorders are associated with excess vulnerability to cardiovascular disease, and possibly some cancers, through accelerated organismal aging. ${ }^{52}$ How complexity loss, interpretable as a marker of degraded system adaptability, has a role in these apparently diverse syndromes (aging, depression, cardiovascular disease) remains to be determined.

Measures of complexity are likely to be useful in complementing, not replacing, current time and frequency domain metrics of HR dynamics. In this study, the mean HR (inversely related to the mean interbeat interval) showed a very small but significant increase (equivalent to 6 beats per min) in the depression versus non-depression groups (Table 3 ). However, of the HRV measures designed to assess cardiac vagal tone modulation, only one, pNN10, was significantly reduced in the depression group. Other measures of cardiac vagal modulation (pNN25 and 50, rMSSD, HF power and LF/HF ratio) were not significantly different. In contrast, VLF and LF power, and SDNNINDX, which were significantly lower in the depression group, do not have a well-established physiologic interpretation. Overall, these HRV results are compatible with decreased vagal and increased sympathetic modulation, as previously described in depression. However, the HRV measures, themselves, are also not entirely self-consistent, which it is not unexpected in light of the range of findings reported in other studies. ${ }^{19-23,47}$

Testing the diagnostic power of the complexity method and/ or of any particular combination of the complexity and traditional HRV methods was outside the scope of our work. This task will require a much larger database than the one probed here.

This study has a number of limitations. First, depression is a heterogeneous syndrome, and multiple sources of variance may exist within patients (for example, severity, typicality, seasonality and number of previous episodes). Second, we excluded patients with more serious forms of psychiatric comorbidity. Third, the sample size and the methods did not allow for analysis by depressive subtypes. Advantages of this study are the restriction to unmedicated subjects with MD, not just anxiety disorder, and the exclusion of those with confounding effects due to comorbidities. Further, we examined HR dynamics not only during the entire sleeping period but also during comparable sleep phases, minimizing activity and sleep stage effects. Future studies are needed to confirm these results in larger populations, including both men and women, and, importantly, to test whether remission is associated with restoration of more complex cardiac interbeat interval dynamics.

\section{Conflict of interest}

The authors declare no conflict of interest.

Acknowledgements. We gratefully acknowledge all the individuals who participated in this study. This research was supported by the Belgian National Fund for Scientific Research (FNRS), which sponsored Dr Samuël Leistedt, The G Harold and Leila Y Mathers Foundation, The James S McDonnell Foundation, the NIHsponsored Research Resource for Complex Physiologic Signals (U01EB008577) and the NIA (K99/R00 1K99AG030677). We also acknowledge the Société Belge Francophone de Médecine du Sommeil.

1. Kessler RC, Berglund P, Demler O, Jin R, Koretz D, Merikangas KR et al. The epidemiology of major depressive disorder: results from the National Comorbidity Survey Replication (NCS-R). JAMA 2003; 289: 3095-3105.

2. Hasin DS, Goodwin RD, Stinson FS, Grant BF. Epidemiology of major depressive disorder: results from the National Epidemiologic Survey on Alcoholism and Related Conditions. Arch Gen Psychiatry 2005; 62: 1097-1106.

3. Murray CJ, Lopez AD. Alternative projections of mortality and disability by cause 1990-2020: Global Burden of Disease Study. Lancet 1997; 349: 1498-1504.

4. Musselman DL, Evans DL, Nemeroff CB. The relationship of depression to cardiovascular disease. Arch Gen Psychiatry 1998; 55: 580-592.

5. Belmaker RH, Agam G. Major depressive disorder. N Engl J Med 2008; 358: 55-68.

6. Penninx BWJH, Beekman ATF, Honig A, Deeg DJH, Schoevers RA, van Eijk JTM et al. Depression and cardiac mortality: results from a community-based longitudinal study. Arch Gen Psychiatry 2001; 58: 221-227.

7. Lesperance F, Frasure-Smith N, Talajic M, Bourassa MG. Five-year risk of cardiac mortality in relation to initial severity and one-year changes in depression symptoms after myocardial infarction. Circulation 2002; 105: 1049-1053.

8. Carney RM, Freedland KE, Miller GE, Jaffe AS. Depression as a risk factor for cardiac mortality and morbidity: a review of potential mechanisms. J Psychosom Res 2002; 53: 897-902.

9. Parissis JT, Fountoulaki K, Filippatos G, Adamopoulos S, Paraskevaidis I, Kremastinos D. Depression in coronary artery disease: novel pathophysiologic mechanisms and therapeutic implications. Int J Cardiol 2007; 116: 153-160.

10. American Psychiatric Association. Diagnostic and Statistical Manual of Mental Disorders, 4th edn, DSM-IV-TR. (text revision). American Psychiatric Association: Washington DC, 2000.

11. Steiger A, Kimura M. Wake and sleep EEG provide biomarkers in depression. J Psychiatr Res 2010; 44: 242-252.

12. Miller $\mathrm{AH}$, Maletic $\mathrm{V}$, Raison $\mathrm{CL}$. Inflammation and its discontents: the role of cytokines in the pathophysiology of major depression. Biol Psychiatry 2009; 65: 732-741.

13. Samuels AM. The brain-heart connection. Circulation 2007; 116: 77-84.

14. Valentini M, Parati G. Variables influencing heart rate. Prog Cardiovasc Dis 2009; 52: $11-19$.

15. Costa M, Goldberger AL, Peng C-K. Multiscale entropy analysis of complex physiologic time series. Phys Rev Lett 2002; 89: 068102-1-068102-4.

16. Chialvo DR. Physiology: unhealthy surprises. Nature 2002; 419: 263.

17. Costa M, Goldberger AL, Peng C-K. Multiscale entropy analysis of biological signals. Phys Rev E Stat Nonlin Soft Matter Phys 2005; 71: 021906-1-18.

18. Yeragani VK. Major depression and long-term heart period variability. Depress Anxiety 2000; 12: 51-52.

19. Yeragani VK, Rao KA, Smitha MR, Pohl RB, Balon R, Srinivasan K. Diminished chaos of heart rate time series in patients with major depression. Biol Psychiatry 2002; 51: 733-744. 
20. Glassman AH, Bigger JT, Gaffney M, Van Zyl LT. Heart rate variability in acute coronary syndrome patients with major depression: influence of sertraline and mood improvement. Arch Gen Psychiatry 2007; 64: 1025-1031.

21. Licht CMM, de Geus EJC, Zitman FG, Hoogendijk WJG, van Dyck R, Penninx BWJH. Association between major depressive disorder and heart rate variability in the Netherlands Study of Depression and Anxiety (NESDA). Arch Gen Psychiatry 2008; 65: 1358-1367.

22. Servant $D$, Logier R, Mouster $Y$, Goudemand M. Heart rate variability. Applications in psychiatry. Encephale 2009; 35: 423-428.

23. Kemp AH, Quintana DS, Gray MA, Felmingham KL, Brown K, Gatt JM. Impact of depression and antidepressant treatment on heart rate variability: a review and metaanalysis. Biol Psychiatry 2010; 67: 1067-1074.

24. Grassberger P. Information and Complexity Measures in Dynamical Systems. In Atmanspacher $\mathrm{H}$, Scheingraber $\mathrm{H}$ (eds). Information Dynamics. Plenum Press: New York, 1991 pp 15-33.

25. Goldberger AL, Giles F. Filley lecture. Complex systems. Proc Am Thorac Soc 2006; 3 467-472.

26. Goldberger AL, Amaral LA, Hausdorff JM, Ivanov PCh, Peng C-K, Stanley HE. Fractal dynamics in physiology: alterations with disease and aging. Proc Natl Acad Sci USA 2002; 19(Suppl 1): 2466-2472

27. Goldberger A, Peng C-K, Lipsitz LA. What is physiologic complexity and how does it change with aging and disease? Neurobiol Aging 2002; 23: 23-26.

28. Norris PR, Anderson SM, Jenkins JM, Williams AE, Morris Jr JA. Heart rate multiscale entropy at three hours predicts hospital mortality in 3,154 trauma patients. Shock 2008; 30 17-22.

29. Riordan Jr WP, Norris PR, Jenkins JM, Morris Jr JA. Early loss of heart rate complexity predicts mortality regardless of mechanism, anatomic location, or severity of injury in 2178 trauma patients. J Surg Res 2009; 156: 283-289.

30. Ferrario M, Signorini MG, Magenes G. Complexity analysis of the fetal heart rate variability: early identification of severe intrauterine growth-restricted fetuses. Med Biol Eng Comput 2009; 47: 911-919.

31. Richman JS, Moorman JR. Physiological time series analysis using approximate entropy and sample entropy. Am J Physiol 2000; 278: H2039-H2049.

32. Pincus SM. Assessing serial irregularity and its implications for health. Ann NY Acad Sci 2001; 954: 245-267.

33. Task Force of the European Society of Cardiology the North American Society of Pacing Electrophysiology. Heart rate variability: standards of measurement, physiological interpretation, and clinical use. Circulation 1996; 93: 1043-1065

34. Hamilton M. A rating scale for depression. J Neurol Neurosurg 1960; 23: 56-62.

35. Buysse DJ, Reynolds CF, Monk TH, Berman SR, Kupfer DJ. The Pittsburgh sleep quality index: a new instrument for psychiatric practice and research. Psychiatry Res 1989; 28 193-213.

36. Leistedt SJ-J, Coumans N, Dumont M, Lanquart J-P, Stam CJ, Linkowski P. Altered sleep brain functional connectivity in acutely depressed patients. Hum Brain Mapp 2009; 30 2207-2219.

37. Agnew Jr HW, Webb WB, Williams RL. The first night effect: an EEG study of sleep. Psychophysiology 1966; 2: 263-266.
38. Rechtschaffen A, Kales A (eds) A manual of standardized terminology techniques and scoring system for sleep stages of human subjects. UCLA Brain Information Service/Brain Research Institute, University of California: Los Angeles, CA, 1968.

39. Moody GB, Mark RG. Development and evaluation of a 2-lead ECG analysis program. Computers in Cardiology 1982; 9: 39-44.

40. Mietus JE, Peng CK, Henry I, Goldsmith RL, Goldberger AL. The pNNx-files: re-examining a widely-used heart rate variability measure. Heart 2002; 88: 378-380.

41. Saul JP, Albrecht $P$, Berger RD, Cohen RJ. Analysis of long term heart rate variability: meth- ods, 1/f scaling and implications. Comput Cardiol 1988; 14: 419-422.

42. Bigger JT, Steinman RC, Rolnitzky LM, Fleiss JL, Albrecht P, Cohen RJ. Power law behavior of RR-interval variability in healthy middle-aged persons, patients with recent acute myocardial infarction, and patients with heart transplants. Circulation 1996; 93: 2142-2151.

43. Kerkhofs M, Linkowski P, Lucas F, Mendlewicz J. Twenty-four-hours patterns of sleep in depression. Sleep 1991; 14: 501-506.

44. Kupfer DJ. Sleep research in depressive illness: clinical implications - a tasting menu. Biol Psychiatry 1995; 38: 391-403.

45. Schulz S, Koschke M, Bar K-J, Voss A. The altered complexity of cardiovascular regulation in depressed patients. Physiol Meas 2010; 31: 303-321.

46. Yang AC, Tsai S-J, Yang C-H, Kuo C-H, Chen T-J, Hong C-J. Reduced physiologic complexity is associated with poor sleep in patients with major depression and primary insomnia. J Affect Disord 2011; 131: 179-185.

47. Boettger S, Hoyer D, Falkenhahn K, Kaatz M, Yeragani VK, Bar K-J. Nonlinear broad band dynamics are less complex in major depression. Bipolar Disord 2008; 10: 276-284.

48. Jokinen V, Syvaënne M, Maëkikallio TH, Airaksinen KEJ, Huikuri HV. Temporal agerelated changes in spectral, fractal and complexity characteristics of heart rate variability. Clin Physiol 2001; 21: 273-281.

49. Lipsitz LA. Aging as a Process of Complexity Loss. In: Deisboeck TS, Kresh JY (eds). Topics in Biomedical Engineering International Book Series: Complex Systems Science in Biomedicine. Springer: New York, NY, 2006, pp 641-654.

50. Starr JM, McGurn B, Harris SE, Whalley LJ, Deary IJ, Shiels PG. Association between telomere length and heart disease in a narrow age cohort of older people. Exp Gerontol 2007; 42: 571-573.

51. Epel ES, Blackburn EH, Lin J, Dhabhar FS, Adler NE, Morrow JD et al. Accelerated telomere shortening in response to life stress. Proc Natl Acad Sci USA 2004; 101: 17312-17315.

52. Simon NM, Smoller JW, McNamara KL, Maser RS, Zalta AK, Pollack MH et al. Telomere shortening and mood disorders: preliminary support for a chronic stress model of accelerating aging. Biol Psychiatry 2006; 60: 432-435.

Translational Psychiatry is an open-access journal published by Nature Publishing Group. This work is licensed under the Creative Commons Attribution-Noncommercial-No Derivative Works 3.0 Unported License. To view a copy of this license, visit http://creativecommons.org/licenses/by-nc-nd/3.0/ 